\title{
Decision Analysis in Management of Information Systems Incidents
}

\author{
D. TCHOFFA ${ }^{1}$, L. DUTA ${ }^{2}$, A. EI MHAMEDI ${ }^{1}$ \\ ${ }^{1}$ Equipe MGSI - Université de Paris8 / LISMMA, \\ 140, rue de la nouvelle France, 93100 Montreuil, FRANCE, \\ d.tchoffa@iut.univ-paris8.fr, a.elmhamedi@iut.univ-paris8.fr \\ ${ }^{2}$ Automation and Computer Science Department, \\ Valahia University of Targoviste, ROMANIA \\ duta@valahia.ro
}

\begin{abstract}
Distributed systems are characterized by heterogeneity and also by multiple applications often interdependent whose programming is done by separate teams with no communications between them. Dysfunctions and problems that occur in these systems have multiple and important consequences. Difficulties encountered during discovering origins and causes of these problems are proportional with user information quality demand. In this paper a method to manage incidents in information systems using decision analysis is proposed.
\end{abstract}

Keywords: Decision analysis, incidents management, case basis reasoning, influence diagrams

\section{Introduction}

In actual economic situation, enterprises must be more effective and innovating in order to create added value for their customers. Pressed by the emergency of results, they are forced to improve the efficiency and the productivity of their employees. Some enterprises cannot undergo the manual and reduce process inefficiency. It is the moment to transform their processes more reactively facing their customers, to improve their efficiency, to reduce the costs and to limit the risks.

While using their best practice and experience in information and processes management, enterprises can adopt a new approach in their work. Thus, treating of industrial system's dysfunctions, incidents (or risk) management process is undergoing and it imposes itself as one that asks for more ingenuity in design and setup of an efficient method to minimize the maintenance costs of industrial applications.

In all sectors of activities, the impact of cascade incidents became one of most elevated budgets. The continuous interest growth for finding efficient dysfunctions management methods is justified by the trained teams, more effective tools of production and huge investments in decision aid tools so as to minimize incidents occurrence.

Using a tool for incidents management allows minimizing delays between treatment phases, to reduce services unavailability, and costs dues to the loss of service. However, methods used for this management are various. In literature, the main idea is to integrate intelligence in incidents management systems, either using a predefined formal set of rules (Rule Based Reasoning - RBR) or set of cases that summarizes and diagnoses previous dysfunctions and gives resolutions (Case Based Reasoning - CBR). A RBR diagnosis system implies a knowledge base that memorizes in a formal language the specific knowledge for the occurred problems and the operators reasoning their current operations. The major inconvenience of a RBR system is that the rules are established only for specific problems and are hardly maintainable (Luger, 2005). A CBR system finds, adapts and reuses old solutions to already met problems to solve new occurred problems or to criticize new solutions.

Taking into account previous considerations, one aims to conceive a tool, not only for incidents statistical analysis, but also to assist operators in finding solutions at the time of the procedures.

Our approach includes automatic incidents treatment and it is close to CBR architecture. A decision aid tool that establishes influences and causes between actions and incidents is presented in this paper.

\section{State of the Art}

Many different methods and models have been proposed in the literature to support efficient incidents investigation in information systems (Hollnagel, 2004; Factor et al., 2007; Santos- 
Reyes and Beard, 2009). As was found in the study of (Rollenhagen et al., 2010) the time invested in specific incidents investigation may be rather limited due to resource constraints. Many studies argue that there is a need for more effective methods to overcome resistance to change, in addition to a need for better methods to explain accidents or incidents which could help to avoid future negative events as the strategies for finding and analysing causes. For an incident investigation agencies include in their policy documents an explicit section on their specific view on their strategies.

In the article (Langevin and al., 2008), authors focuses on the need to integrate the aspects related to the human activity.

The use of human factors approach in the design of process control systems throughout the industry presents many opportunities for improvements with regards to systems efficiency, effectiveness, reliability and safety. Contribution of human factors issues, in the higher number of incidents and accidents represent about one third of and factors and can be attributed to the design of the technical system itself (Manning, and al., 2004).

There is a need for unifying what is separately done in engineering, human factors and organization science. The Orchestra model (Boy, 2009) will support the identification of both deliberate and emergent cognitive functions during the life cycle of a multi agent life-critical system. It is based on previous work on cognitive function analysis (Boy, 2011) and function allocation work (Grote et al., 2000.)

This will require advanced concepts and technologies, along with higher levels of automation; all of which will result in changes to roles and responsibilities for employees and managers. These transitions, in combination with increased interaction with automation, can lead to unwanted side effects, such as increased errors, loss of situational awareness, or mode confusion. This necessitates an understanding of human factors, which studies how humans' abilities, characteristics, and limitations interact with the design of the equipment they use, the environment in which they function, and the jobs they perform (Hollnagel, 2009).

Industries undertake human factors research as part of their incident management systems- related programs and projects to assess the envelope of human performance capabilities and limitations with new concepts and automation. Decision analysis on information systems incidents also highlights necessity of collaborations and best practices for coordinating research activities and identifying opportunities for joint work. The key challenges identify important, complex issues that pose significant technical risk in realizing the full benefits of management incidents in industries (Tchoffa, et al, 2012 a).

\section{Incidents Causes Analysis}

In industry, an incident is represented by all events that are not part of the standard service working and that can cause an interruption or a reduction of the service quality (Tchoffa and El Mhamedi, 2012 b) and other considerable damages (Grangier, 2006).

In the second chapter of their book, (Kossiakoff et al., 2011) explored the full spectrum of systems complexity and the reason why the systems engineering landscape presents a challenge for systems engineers. To illustrate the types of systems that fit within the above definition, examples of Signal and Data Systems and Material and Energy Systems list ten modern systems and their principal inputs, processes, and outputs. An automobile case is also cited as an example of a large class of systems that require active interaction (control) by a human operator.

Boy, G. A., developed Human-centred design for different reasons and issues: "We all need to understand what we want to do with technology, how we should organize ourselves to a better life and finally find out whom we are and have become” (Boy, 2012).

The causes of incidents can be classified as it follows (Reason, 2004):

\subsection{Technical causes}

Industrial workshops are served by more and more sophisticated equipment. The equipment failure can be caused either by a computer, an electric breakdown, a mechanical or abnormal functioning of an automaton (Hollnagel and Woods, 2005).

The allocation of redundant components can affect other non-functional quality attributes of the system, which are as important as reliability 
and very often conflicting with each other, such as cost and response time (Meedeniya, I. and al., 2009).

\subsection{Human errors}

A cognitive system, and therefore also a joint cognitive system, is defined by its ability to maintain control, or more specifically to modify its behaviour on the basis of experience so as to achieve specific anti-entropic ends (Hollnagel and Woods, 2005).

Human errors are important causes in failure of industrial systems. (Reason, 2004) distinguishes three types of mistakes in an industrial enterprise:

- Routine error corresponds to the work based on routine procedures. It is an error occurring during the surveillance of a task execution. That means that the operator didn't become aware of the failure occurrence. This is a frequent problem in manufacturing process.

- Knowledge activation error. In this case the operator has difficulties met when solving a problem. He is conscious of the problem and he looks for a solution. The error is going to result from a bad (or the activation of a bad) rule.

- Knowledge possession error where the operator ignores the solution of the problem that has to be adjusted. He mobilizes all his reflection slowly to produce a new solution. The error provides a good solution out of time or a bad solution.

\subsection{Organizational causes}

The organizational error causes can be found in several circumstances like the occurrence of an incident in particular processes, capacity saturation of the industrial enterprise, increase of activities flow, maladjustment between the growing needs and organization capacity, unexpected interaction between processes in rare circumstances (Figure 1).

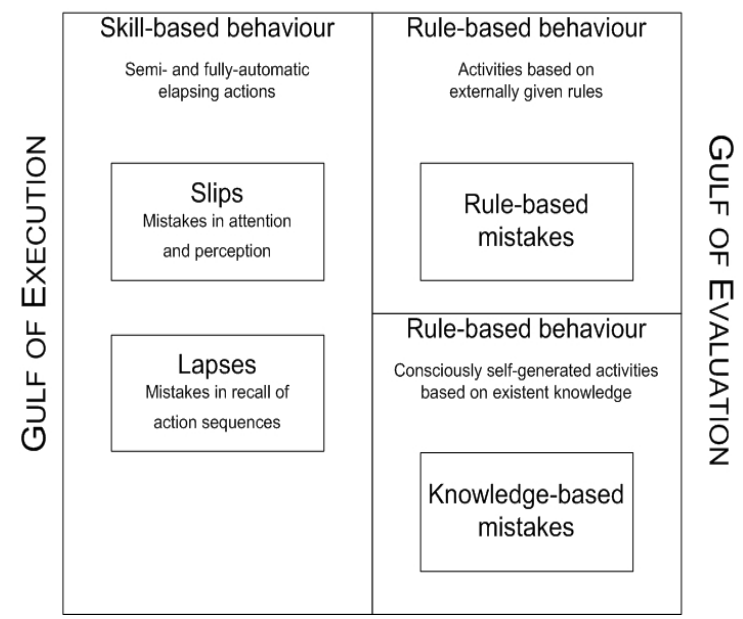

Figure 1. Classification of mistakes (after Reason, 2004)

Reason identifies different sources of organization failure. As it is shown in Figure 2, these can be connected to risking procedures, to internal organization or qualifications.

Measuring safety is generally considered a very difficult task, at least compared with many other aspects of measuring the results of changes in organizations (Lofquist et al., 2011).

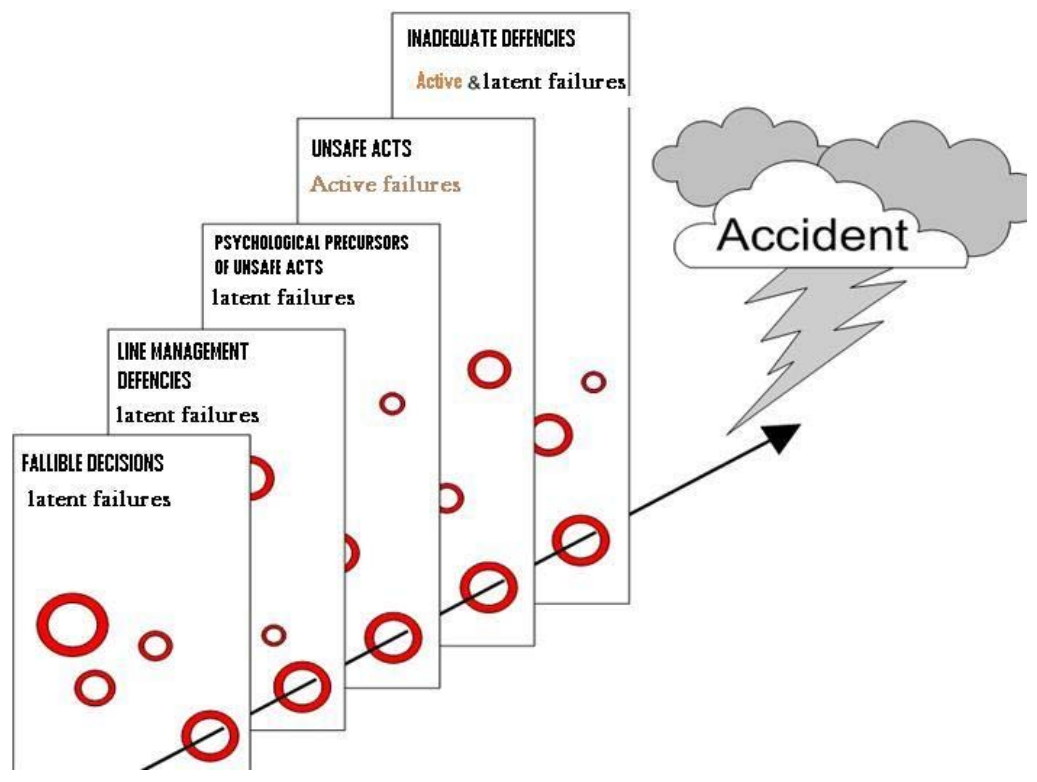

Figure 2. How errors lead to accidents (after Reason, 2004). 
In 2002, human error was credited with $15 \%$ of unplanned outages (Margeson, 2003). In 2007, human beings were credited with 40 per cent of unplanned outages (Morrill al., 2008).

Nowadays, the view of Swiss Cheese Model as presented by Reason is discussed by those who consider diversity. Most manuals furthermore included the information as a central aspect, something that Leveson (2004) has pointed as at central in many current systems. In other words, models of "human error" and organizational failures must be complemented by something that could be called sociotechnical or systematic accident models (Hollnagel, E., 2004).

In his latest book, Erik Hollnagel describes his research in FRAM analysis method highlighting the model of socio-technical systems in the global view of Human Factor in information systems (Hollnagel, E., 2012).

\section{Incidents Management}

The goal of incidents management is to create the premises for resuming as soon possible the normal working of a service, to minimize the negative impact on the activities and to ensure quality improvement and the availability of services (O`Callaghan and Mariappanadar, 2010).

A Human Reliability Analysis (HRA) methodology supports other HFE technical elements and the Probabilistic Risk Assessment (PRA). One source of human actions considered in the HRA analysis is the Human Factors Engineering (HFE) Issues Tracking System (HFEITS), which tracks any identified HFE issues and human engineering discrepancies (HEDs) throughout the project life cycle (NRC, 2010).

In 2007, the Skype service remained inaccessible during two days because of a software bug which show that Internet phone cannot replace stationary telephone definitely (Rodriguez and Druschel, 2010).

Managing industrial incidents facilitates resolution of problems, issues, and human engineering discrepancies by providing a means to record and track issues throughout the process of design, development, and evaluation (O'Hara and al., 2008 and NRC, 2007). Incidents management process consists in detecting and recording incidents data base, to classify and diagnose them, in order to understand their causes, to make a follow-up of their resolution and service restoration (Metzger, 2003). Incidents management process must allow a best understanding of roles and responsibilities of intervening actors, a high speed of execution at the time of an incident, an optimal resources management, best communication between parts and integrated human factors principles, methodologies, guidelines in the design and planning (Gody, 2011).

Figure 3 details the different stages of the incident management. Phases of incidents management are:

- The first stage consists in detecting the incident, either following a call user, either via tools of surveillance. After the detection it is necessary to record the incident. During the registration it is necessary to complete the information.

- Classification consists in classifying the incident according to his valued reason in order to identify the group of support. The classification must permit to define the incident priority and the gravity.

- Investigation and diagnosis is performed to find the cause of the incident, and so to identify the requisite solution

- Solving and restoring the service. The solution is temporary or definitive, the goal being to restore the service as soon as possible.

- The last stage consists in verifying the effective restoration of the service, that all information relative to the incident is registered.

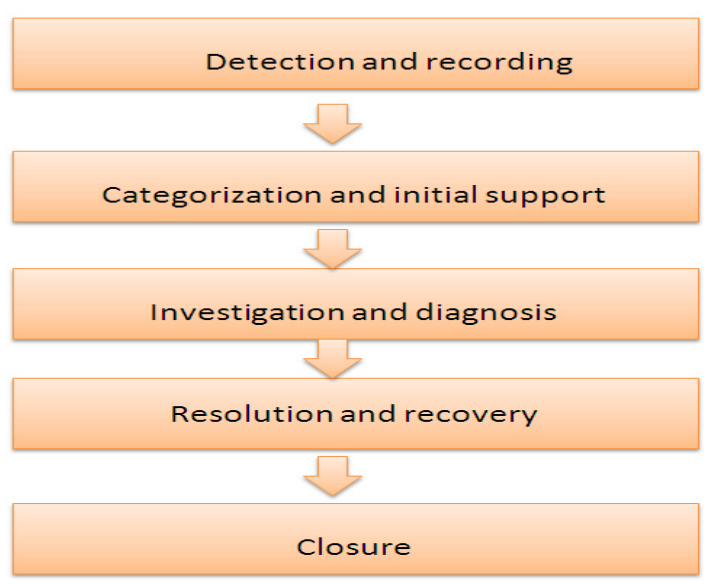

Figure 3. Phases of incident management 


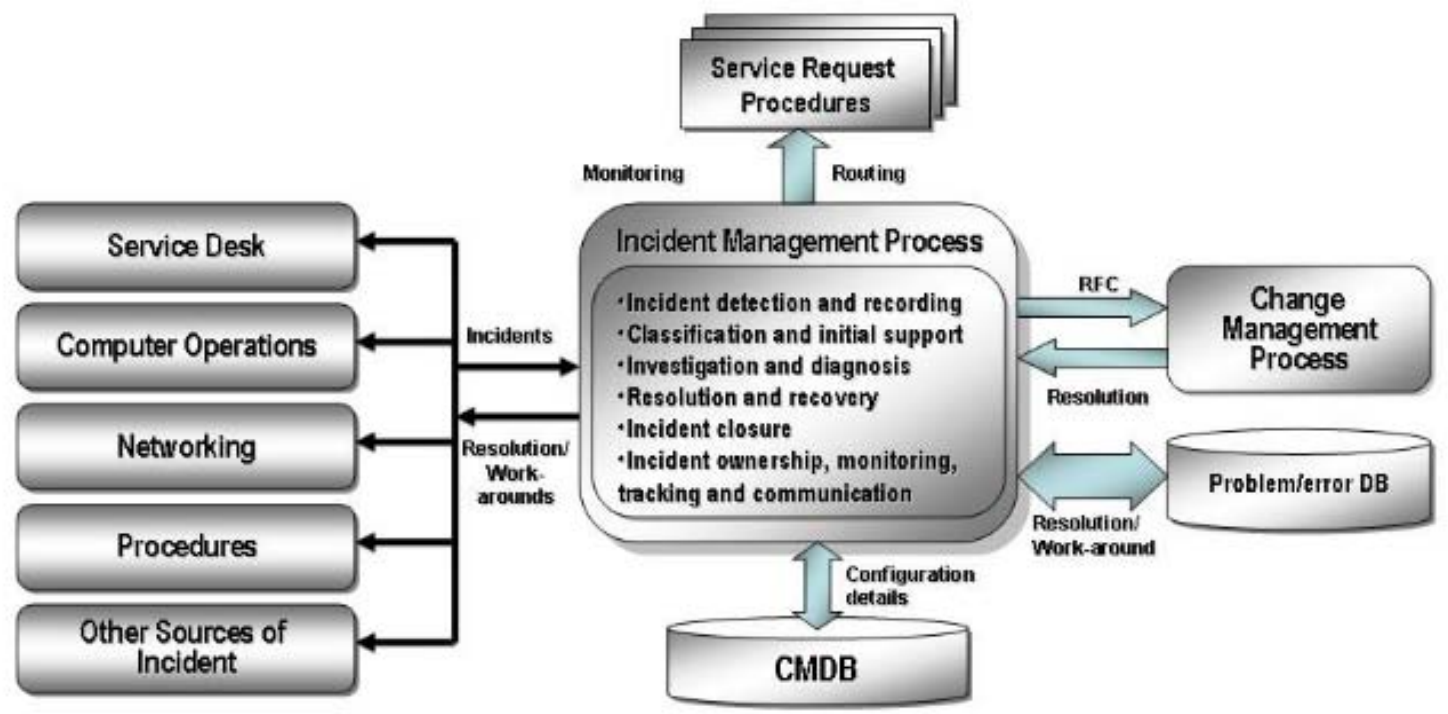

Figure 4. Description of the incidents management process (from ITIL, 2007)

In an incident management process there are input and output elements. In (ITIL, 2007) the input elements are (Figure 4):

- incidents details

- relations between incidents and known problems

- resolution of an incident

- change demands in incident correction

In exchange, the output elements are:

- demands to solve incidents

- data base updating

- users communication

- hierarchy reporting

\section{Case Study}

To illustrate our application, we have used the Renault incidents management data base exemplified in the thesis of (Tchoffa, 2006). This data base for bugs, incidents, and configuration changes allows managing information system incidents of the Renault enterprise.

The system shows how an incident should be treated, its description, its impact on the information system, its critical degree, the brought corrections, observations registered after correction accomplishment, the concerned elements of configurations solving and enclosing data of the incident (Figure 5).

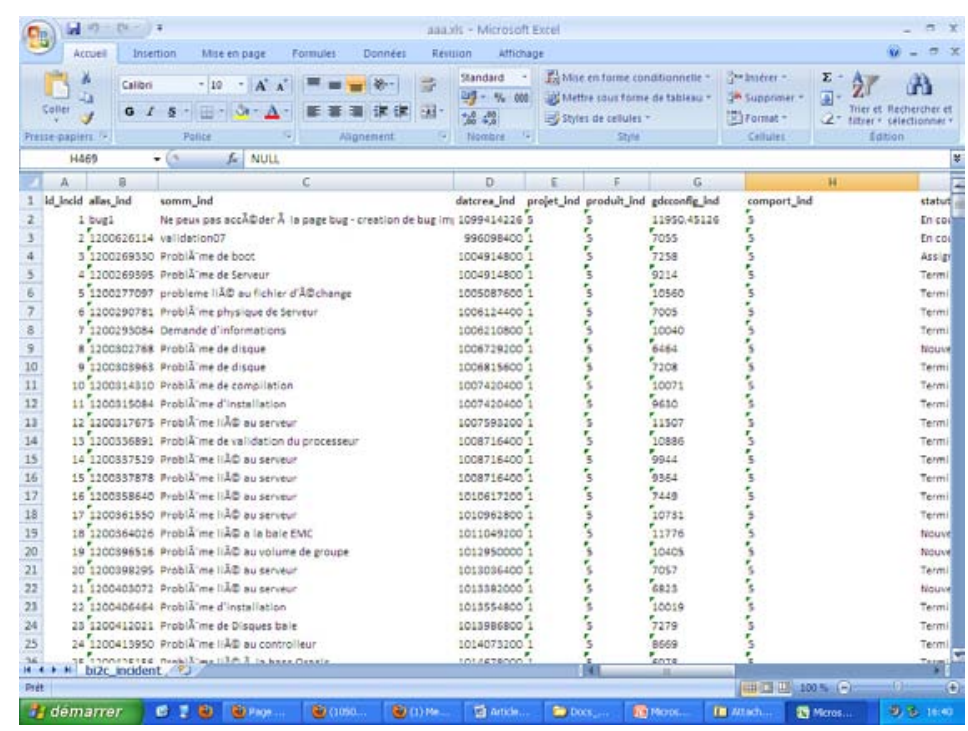

Figure 5. Renault incident data base 
We exploited only a part of the incidents data base, limiting to hundred incidents recorded during the year 2005, and to the eight main cause failures: server errors, instruction errors, disk errors, boot errors, installation errors, file errors, memory errors and network errors. The information system is failing if one of these problems occurs and is followed up by a serious disruption of the system.

The aim of analysis is to discover the relation between failure causes and effects and between causes themselves. First the incidents data base is reorganized according to failure causes. Then existing relationships are determined.

The proposed approach has been implemented with the help of Decision Programming Language (DPL ${ }^{\circledR}$ 7.0), an application for decision analysis, created by ADA Inc. and Microsoft Corporation.

\section{Decision Analysis using DPL $®$}

There is a plethora of decision analysis methods which can be found and utilized in decision analysis (Filip, 2002). The proposed approach has been implemented with the help of Decision Programming Language (DPL 7.0), a software tool for decision analysis, created by ADA Inc. and Microsoft Corporation. It can be included in the class of decision support systems ( Filip, 2008)

DPL 7.0 (Palisade, 2012) is a suite of applications that allows editing decision trees as well as drawing influence diagrams, rainbow diagrams, Vann diagrams, Tornado diagrams, and it provides a complete decision analysis by evaluating the risk profile and utility of decisions. It enables performing sensitivity analysis by spread-sheeting the decision trees and performing the Monte-Carlo simulation. For decision analysis runs, DPL automatically displays risk profiles as cumulative probability distributions. DPL has the facility to link a model with a spreadsheet.

When the analysis is run, DPL will proceed along each tree path, updating cells in the spreadsheet and asking for calculated values back whenever necessary. At end of each path, DPL will calculate the endpoint value, then starts down another path, again changing cells in the spreadsheet as it goes. The spreadsheet will be updated and recalculated many times during the analysis.

DPL also allows to running optimization methods like Expression Optimization, Series Optimization, and Lottery Optimization.

\section{Implementation}

An influence diagram is drawn using previous spreadsheets data. In Figure 6, relations between failure causes and decisions to solve them are represented. One notes that failures are caused mainly by installation errors, while instruction errors can provide disk or server errors.

In Figure 6 one the following notations are used:

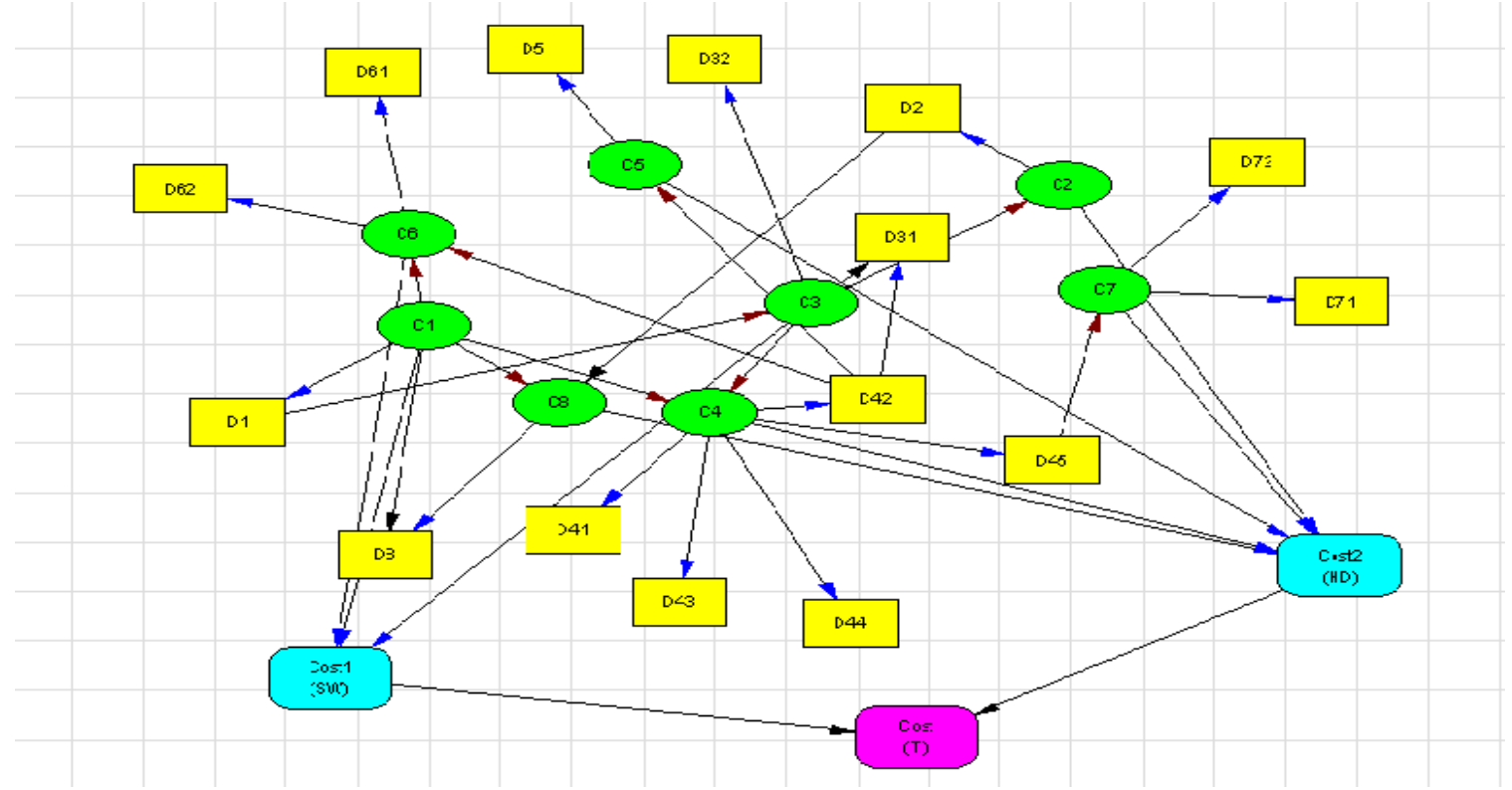

Figure 6. Influence diagram for incidents causes. 
Chance nodes (incidents events):

- C1 installation errors (occurrence 35\%)

- C2 disk errors (10\%)

- C3 instruction errors (17\% )

- C4network errors (10\%)

- C5server errors (8\%)

- C6 boot errors (5\% )

- $\quad$ C7 memory errors (5\%)

- C8 file errors (10\%)

Decision nodes (solutions that solve the incident)

- D1 software reinstalling

- D2 disk replacement and data reloading

- D31 documentation providing

- D32 correction of the incorrect script

- D41 upgrading vxfs version

- D42 system disk exchange

- D43 dump analyze

- D44 patch installation

- D45 server restarting

- D5 IP address problem solving

- D61 CPU exchange

- D62 firmware update

- D71 memory card exchange

- D72 processor exchange

- D8 files reloading

There are influences and relations between failure causes. For example C1 influences C4, C6 and C8, or C3 influences C2 and C4. On the other hand, the resolution of an incident can influence another incident occurrence with a certain degree of probability. For example D42 can influence the occurrence of C6 or D1 can influence the occurrence of $\mathrm{C} 3$ and so on.

Costs are represented by value nodes. Costs are classified as it follows: SW Costs - induced by software failures and HD Costs induced by hardware failures.

Total cost is calculated as the sum of previous costs plus human errors costs. The aim of the decisional analysis is to find the critical incidents and the best decisions to take so as to minimize the failures costs.

On this model, a complete decision analysis has been performed in four phases.

\subsection{Policy tree}

A DPL Policy Tree shows all possible paths through the tree and indicates the value of all get/pay expressions in the model, the probabilities associated with each chance event outcome, and the rollback values for each node. DPL indicates the optimal alternative for a decision by drawing the branch for the alternative in a darker line. If the decision analysis is run, one obtains the complete decision tree as it is shown in Figures 7 and 8.

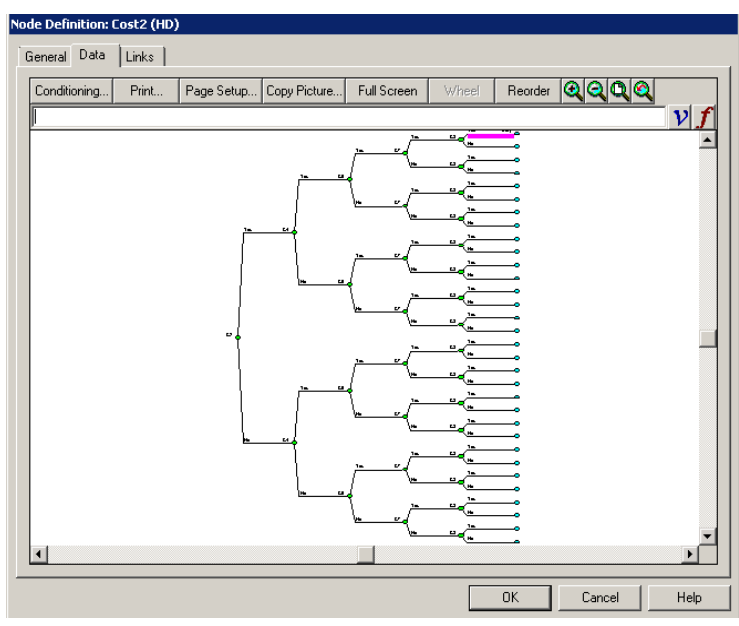

Figure 7. Decision tree

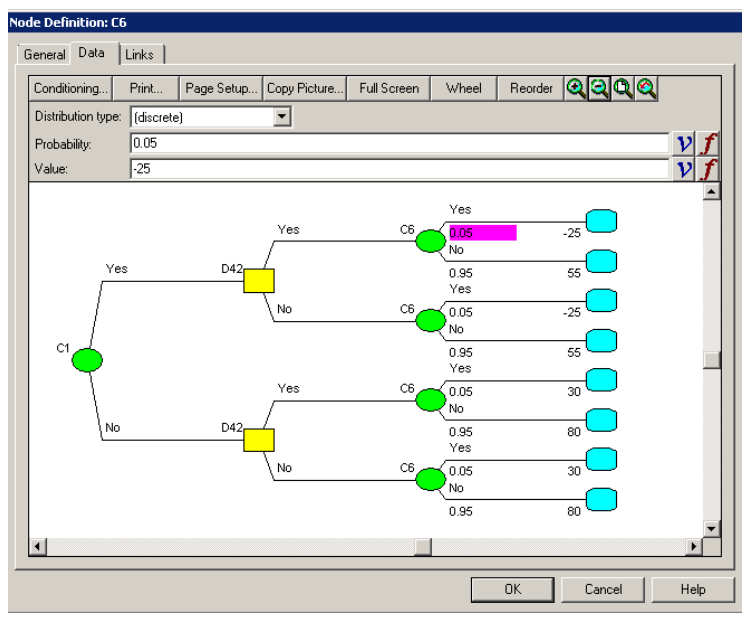

Figure 8. Decision Tree (detail)

\subsection{Policy summary}

Policy Summaries can be generated during the decision analysis. They display the probability distributions for the events in a model given that the optimal policy is followed (referred to as policy-dependent probabilities). For each event, DPL displays the event's states plus an additional state called "does not occur".

The probability indicated for a state is the probability of the state conditioned on the optimal policy. The "does not occur" state indicates the 
probability that the event is not encountered under the optimal policy. This may happen in asymmetric trees, where the same events do not occur on all paths through the tree.

The Policy Summary is particularly useful when a decision occurs fairly late in a large tree. The decision may appear several times in the policy, making it hard to quickly see whether the decision depends on the preceding events or if there is always (or usually) one dominant alternative.

With the Policy Summary, one can quickly tell to what extent downstream decisions are dependent on the preceding events (Figure 9)

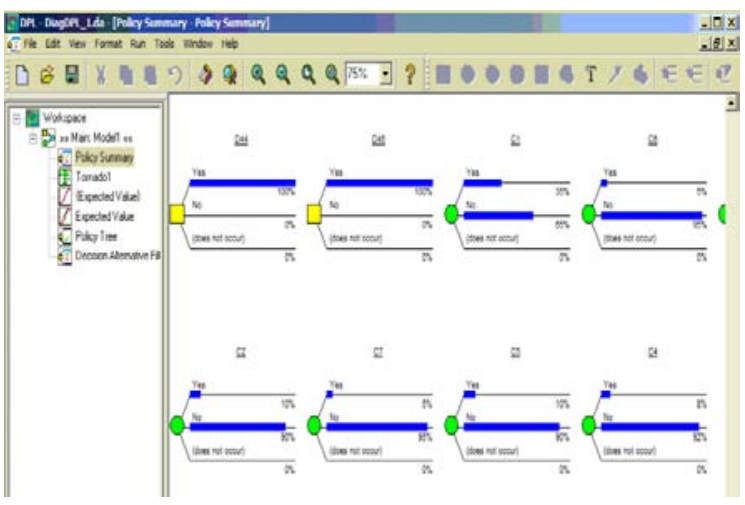

Figure 9. Policy summary

For chance nodes whose probabilities are not conditional, the probabilities of each state occurring will be the same as those entered in the influence diagram unless the event is not encountered in some paths through the tree. For example one of results for our study case is that the processor exchange (D72) must be the last decision to take.

\subsection{Risk profile}

The Risk Profile Chart is the means of displaying the Risk Profile Data. Each Risk Profile Data set is a set of points that represents the range of outcome values under a particular decision policy. The default Risk Profile Data generated is for the expected value of the Objective Function under the optimal policy (best alternative) only.

For example, in the Figure 10 the higher risk is given by an Expected value of 300 unities. The optimal expected (the minimal cost) value is about 240 unities.

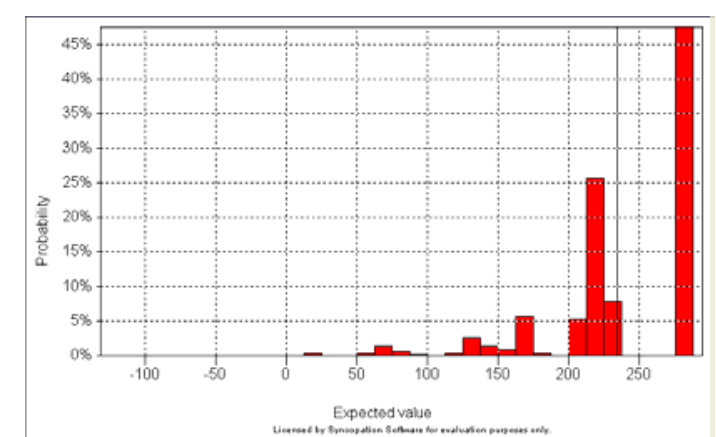

Figure 10. Risk profile chart

\subsection{Sensitivity analysis}

The last phase is to run an analysis to see how sensitive the optimal decision to variables and probabilities changes is.

Tornado Diagrams are most useful with probabilistic models where it is important to incorporate probabilities into the sensitivity analysis. The probability distributions vary widely among chance nodes and the Tornado diagram shows the impact of an incident on the entire model. (Figure 11)

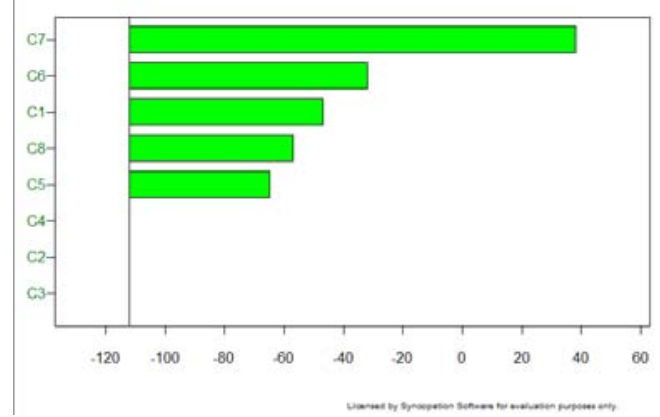

Figure 11. Tornado diagram

The width of the bar for each expected value is the difference between the two runs of the objective function. The bars indicate changes in the optimal policy through color changes. Green indicates the base result optimal policy

One can notice that the chance event C7 has the highest impact on the decision policy. This means that memory errors have the highest influence on the system's errors.

At the end of simulation, the model can be compiled so as to generate a program in the DPL own programming language. This is useful for exporting data and instructions that can be used in other programs. 


\section{Conclusions}

This paper proposes a method for information system incidents management. The approach is based on a complete decision analysis using DPL ${ }^{\circledR}$ software.

Influence diagrams are used to establish influences between failure causes and further solving decisions. Data is imported from a knowledge base. Although most enterprises update their data or knowledge, these are often incomplete at time of decision making. In these cases, experts are consulted to analyze the problem and to take decisions.

Using influence diagrams, one can deduce relations between various failure causes and the impact of these failures on the enterprise information system. The proposed decision analysis allows estimating failures impact on costs and resolution risks.

The next stage in management of information systems incidents is to integrate the previous decision analysis results in prevention model.

\section{REFERENCES}

1. BOY, G. A., The Orchestra: A Conceptual Model for Function Allocation and Scenario based Engineering in Multi-Agent SafetyCritical Systems, Proceedings of the European Conference on Cognitive Ergonomics, Finland, 2009, pp. 187 - 193

2. BOY, G. A., Handbook of HumanMachine Interaction: A HumanCentered Design Approach. Ashgate, Eds, 2011, U.K

3. BOY, G. A., What do we mean by Human-Centered Design of Life-Critical Systems, IOS Press, 2012.

4. DHILLON, B. S., Safety and Human Error in Engineering Systems. CRC Press, Taylor and Francis Group, Boca Raton, 2012

5. FACTOR, R., D. MAHALEL, G. YAIR, The social accident: a theoretical model and a research agenda for studying the influence of social and cultural characteristics on motor vehicle accidents, Accident Analysis and prevention, vol. 39(5), 2007, pp. 914-921.
6. FILIP, F. G., Decizie asistata de calculator: decizii, decidenti , metode si instrumente de baza. Ed. Expert si Ed. Tehnica, Bucuresti, 2002

7. FILIP, F. G., Decision Support and Control for Large-Scale Complex Systems. Annual Reviews in Control, vol. 32(1), 2008, pp. 61-70

8. GODY, A., Human-Machine Interface. PCSR - Sub-chapter 18.1, UKEPR-0002181 Issue 05, 2011.

9. GRANGIER, E., Le bug: une esthétique de l'accident. $\mathrm{PhD}$ Thesis, Université Paris 1 Panthéon - Sorbonne, 2006.

10. GROTE, G., C. RYSER, T. WÄFLER, A. WINDISCHER, S. WEIK, KOMPASS: A method for complementary function allocation in automated work systems, International Journal of Human-Computer Studies, vol. 52, 2000, pp. 267-287.

11. HOLLNAGEL, E., Barriers and Accident Prevention. Ashgate Eds, Hampshire Burlington, England, 2004.

12. HOLLNAGEL, E., D. D. WOODS, Joint Cognitive Systems: Foundations of Cognitive Systems Engineering. CRC Press, Boca Raton, FL, 2005.

13. HOLLNAGEL, E., The Four Cornerstones of Resilience Engineering. In: Nemeth, C., Hollnagel, E., Dekker, S. (Eds.), Resilience engineering perspectives, vol. 2. Preparation and restoration. Ashgate, Farnham, UK, 2009, pp. 117-133.

14. ITIL, Great Britain, London, The Stationery Office. 2007.

15. KOSSIAKOFF, A., W. N. SWEET, S. J. SEYMOUR, S. M. BIEMER, Systems Engineering - Principles and Practice Wiley, 2011, pp. 11-12.

16. LANGEVIN, S., B. JOSEPH, J. BRENKLE, S. STRAUSSBERGER, B. GUIOST, J-Y. LANTES, G. BOY, B. N'KAOUA, Human-centered Design Methodology: An Example of Application with UAVS Mission, Proceedings of the first conference on Humans Operating Unmanned Systems (HUMOUS’08), Brest, France, 2008. 
17. LeVeson, N., A New Accident Model for Engineering Safe Systems, Safety science no. 42, 2004, pp. 237-270.

18. LOFQUIST, E. A., A. GREVE, U. H. OLSSON, Modeling Attitudes and Perceptions as Predictors for Changing Safety Margins during Organizational Change. Safety Science, vol. 49(3), 2011, pp. 531-541.

19. MANNING, S, D., C. E. RASH, P. A. LeDUC, R. K. NOBACK, J. McKEON, The Role of Human Causal Factors in U.S. Army Unmanned Aerial Vehicle Accidents (DOT/FAA/AM-04/24). Technical report. Federal Aviation Administration, Oklahoma City. 2004.

20. MARGESON, B. The Human Side of Data Loss. Disaster Recovery Journal, vol. 16(2), 2003, pp. 48-48.

21. MARLING, C. R., G. J. PETOT, L. S. STERLING, Integrating Case-Based and Rule-Based Reasoning to Meet Multiple Design Constraints. Computational Intelligence, vol. 15(3), 1999, pp. 308-332.

22. MEEDENIYA I., A. ALETI, B. BUHNOVA, Redundancy Allocation in Automotive Systems using Multiobjective Optimization. In Symposium of Avionics/Automotive Systems Engineering (SAASE’09), San Diego, CA, 2009.

23. METZGER, R. C., Debugging by Thinking: A Multidisciplinary Approach, DigitalPress, 2003.

24. MORRILL, H., M. BEARD, D. CLITHEROW, Achieving Continuous Availability of IBM Systems Infrastructures. IBM Systems Journal, vol. 47(4), 2008, pp. 493-503.

25. NRC (2007 and 2010). Standard Review Plan, Chapter 18 - Human Factors Engineering (NUREG-0800) and Human Factors Engineering Program Management Plan (NP-TR-0610-290-NP). Washington, DC: U.S. Nuclear Regulatory Commission.

26. SANTOS-REYES, J., A. N. BEARD, A Systemic Analysis of the Edge Hill Railway Accident. Accid. Anal. Prev. 41(6), 2009, pp. 1133-1144.

27. O‘CALLAGHAN, K., S. MARIAPPANADAR, Incident Manager. Meet PHiL CROSS. IT
Service Management Forum Australia, Informed Intelligence, 2010, p. 7.

28. O'HARA, J., J. HIGGINS, W. BROWN, J. PERSENSKY, P. LEWIS, J. KRAMER, A. SZABO, M. BOGGI, Human Factors Considerations with Respect to Emerging Technology in Nuclear Power Plants (NUREG/CR-6947). Washington, DC: U.S. Nuclear Regulatory Commission 2008.

29. Palisade, Software for risk and decision analysis (www.palisade.com, accessed on 07.12.2012)

30. REASON, J., Managing the Risks of Organizational Accidents. Proceedings of The 5th Risk Management Conference RMC 2004, Cleveland, October 27, 2004.

31. RODRIGUES, R., P. DRUSCHEL, Peer to Peer Systems, Communications of the ACM, vol. 53(10), 2010, p. 81.

32. ROLLENHAGEN, C. J. W., J. LUNDBERG, E. HOLLNAGEL, The Context and Habits of Accident Investigation Practices: A Study of 108 Swedish Investigators. Safety Science, vol. 48(7), 2010, pp. 859-867.

33. TCHOFFA, D., A partir d'une étude ethno-méthodologique du «bug de l'an 2000", Intégration d'un processus d'amélioration continue de gestion des bugs, incidents, problèmes, changements et configurations pour les systèmes d'informations critiques. Thèse de doctorat, Université Paris 8 St-Denis, 2006.

34. TCHOFFA. D., L. DUTA, A. El MHAMEDI, Decision analysis in Management of Industrial Incidents, Proceedings of the 14th IFAC Symposium on Information Control Problems in Manufacturing, Bucharest, Romania, 2012, pp 951-955.

35. TCHOFFA, D., A. El MHAMEDI, A Decision Aid Tool in Software Maintenance, Proceedings of the 14th IFAC Symposium on Information Control Problems in Manufacturing, Bucharest, Romania, 2012, pp. 946-950. 